\title{
Designing Wearable Systems for Sports: A Review of Trends and Opportunities in Human-Computer Interaction
}

\author{
Eleonora Mencarini, Amon Rapp ${ }^{\circledR}$, Lia Tirabeni, and Massimo Zancanaro
}

\begin{abstract}
This paper presents a literature review of humancomputer interaction works on wearable systems for sports. We selected a corpus of 57 papers and analyzed them through the grounded theory for literature review approach. We identified five themes across the papers: the different research perspectives, the type of sports and sportspeople, the roles of wearables in sports, their wearability, and the different types of feedback. These themes helped us in delineating opportunities for future research: the investigation of different form factors and types of feedback; the consideration of different sportspeople and collaborative tasks; the need of pushing the boundaries of the sports domain; the exploration of the evolution of sports; the interconnection of different devices; and the increase of methodological rigor.
\end{abstract}

Index Terms-Athletes, fitness, sports, systematic literature review, wearable devices, wearable technologies.

\section{INTRODUCTION}

$\mathbf{S}$ PORTS provides interesting opportunities for interactive technologies [1]. The market of wearable devices for sports is currently booming, driven by sport watches (e.g., Suunto, Garmin, Polar), sports apparel (e.g., Nike), IT (e.g., Apple), and emergent wearable products (e.g., Jawbone) manufacturers and companies. The increasing diffusion of these kinds of devices, on the one hand, is the result of the progressive miniaturization of sensors and the consequent improvement of their wearability, a term that refers to the ability of an artifact to be worn on the human body and to adapt to its shape [2]. On the other hand, it is due to the advancements in research on body sensor networks [3] and human activity recognition using wearable sensors [4][6], which allowed for the collection of accurate information

Manuscript received December 7, 2018; revised March 20, 2019; accepted May 18, 2019. This work was supported in part by the Fondazione Caritro grant Prot. SG 1939/17-Rif. Int. 2017.0333 "CASM - Comunicazione Aumentata per l'Apprendimento degli Sport di Montagna. This paper was recommended by Associate Editor F. Giancarlo. (Corresponding author: Amon Rapp.)

E. Mencarini is with the Fondazione Bruno Kessler, 38123 Trento, Italy (e-mail: mencarini@fbk.eu).

A. Rapp is with the Department of Computer Science and the ICxT - ICT and Innovation for Society and Territory, University of Torino, 10149 Torino, Italy (e-mail: amon.rapp@gmail.com).

L. Tirabeni is with the Department of Cultures, Politics and Society, University of Torino, 10153 Torino, Italy (e-mail: lia.tirabeni@unito.it).

M. Zancanaro is with the Department of Psychology and Cognitive Science, University of Trento, 38068 Rovereto TN, Italy, and Fondazione Bruno Kessler, 38123 Trento, Italy (e-mail: massimo.zancanaro@unitn.it).

Color versions of one or more of the figures in this paper are available online at http://ieeexplore.ieee.org.

Digital Object Identifier 10.1109/THMS.2019.2919702 on people's activities and behaviors. This enabled athletes to continuously monitor a variety of parameters in sports as diverse as horse riding [7], badminton [8], basketball [9], rowing [10], and swimming [11].

As wearables for sports are being used by a growing user base, academic research has started focusing on the "human aspects" of such technologies, in order to understand their impacts on athletes' performances and develop more effective ways of interaction. In particular, the human-computer interaction (HCI) community has showed an increasing interest in studying wearables in the sports domain [12]: athletes have idiosyncratic needs that have to be understood in depth in order to create technologies capable of being integrated into their situated practices. Considering the increasing number of HCI studies on wearables for sports, we deem timely to conduct a literature review of this research domain, as reviews are critical to strengthening a field of study [13]. In doing so, we aim to map the topics that have been investigated and the research methods that have been adopted so far, focusing on the human side of interaction. Moreover, we want to start delineating what future strands of research could be undertaken by the HCI community.

Following similar works in the HCI field [14], we adopted the grounded theory for literature review (GTLR) method [15], in which reviewing becomes a rigorous process of searching, selecting, and analyzing studies. Our analysis identifies five relevant themes across the selected papers. The first theme concerns the research perspective, which can be driven by a specific technological opportunity, by a design intent, or by the need to understand how a wearable device can be integrated into situated practices. The second theme relates to the type of sports and sportspeople, highlighting the predominance of individual sports, as well as the focus on a "generic" kind of athlete. The third theme explores the roles that wearables play in sport, that is enabling, improving, or augmenting the sports experience, whereby each role may affect either the physical, cognitive, or social dimension of such experience. The fourth theme addresses wearability, highlighting that current research mostly leverages wrist-worn technologies. The last theme concerns the type of feedback given to the user: in the majority of cases, a real-time feedback is given to athletes or coaches. The analysis of these themes may suggest future directions for research, by deepening and at the same time widening the research focus on wearables for sports: most of the papers we reviewed present preliminary studies from a HCI perspective, as the number of users involved 
is limited, and attention is paid more to technology than to human needs. Given these premises, we delineated six directions for evolving HCI research in this area.

In sum, this review contributes to the HCI field by surfacing a tentative map of HCI studies on wearables for sports, from their first appearance to 2018, and by identifying possible future directions of research based on the gaps identified. This paper is organized as follows: Section II outlines the methodology used. Section III presents the themes identified during the analysis, while Section IV discusses the findings and proposes lines of research to be developed in the future.

\section{Methodology}

In order to analyze the HCI literature on wearables for sports, we employed the Grounded Theory Literature Review [15]. This method is rooted in grounded theory [16], an approach developed in social sciences that aims to build theories through an inductive process of data gathering and analysis. What distinguishes grounded theory from other approaches is an inductive, rather than a hypothetical-deductive stance. An analysis based on grounded theory involves three iterative main stages: 1) collecting and coding data; 2) developing, checking, and integrating theoretical categories; and 3) analyzing and comparing concepts to inductively construct a theory [17]. Accordingly, grounded theory literature review uses the content of the articles under analysis as empirical material to be analyzed in order to build theory through review. As the original grounded theory approach, this review method is based on different stages of analysis to be applied inductively and iteratively [15]: 1) Define: choosing the criteria for including/excluding the papers, identifying the fields of research, determining the appropriate sources, and deciding the specific search terms; 2) Search: collecting the papers that fit in the criteria across the identified sources; 3) Select: refining the sample by manually checking the papers; 4) Analyze: adopting open, axial, and selective coding techniques; and 5) Present: structuring, presenting, and discussing the content of the analysis.

\section{A. Search Through the Identified Sources}

We defined the focus of this review by limiting it to HCI research on wearable devices for sports. In doing so, we excluded those studies exclusively focusing on human activity recognition issues, as well as those investigating wearables for monitoring health. On the one hand, human activity recognition works typically focus on the algorithms proposed, rather than on the human aspects of interaction, and the data visualizations they offer are not intentionally and primarily designed for being interacted by end users (e.g., athletes, coaches) in order to satisfy their situated needs. Conversely, our focus was on studies presenting interfaces purposefully addressed to such end-users. On the other hand, wearables for monitoring health deal with different issues and goals with reference to wearables for sports: while the former concern topics like the patient's adherence to a treatment or her recovery from an injury, the latter are addressed to give support during workouts and races, or to increase the athlete's performance. Although there are applications in the health domain that are tightly related to the sports context, such as physical rehabilitation and health behavior change, we preferred to focus on a narrower area at this stage. This would lead to clearer objectives and better circumscribed themes resulting from the analysis. Moreover, we excluded any sports technology beyond wearable devices, e.g., solutions involving ambient intelligence or tangible interaction.

The papers in this review were collected in June 2018. We chose Scopus and the Association for Computing Machinery Digital Library (ACM DL) as sources as they are the main repositories for HCI conference proceedings and journal articles. We conducted a search in both repositories by using the following terms and connectors for the query: [("sport" or "fitness" or "workout" or "training" or "athlete") and ("wear*")]. As for Scopus, we limited our search to the articles belonging to the following research fields: engineering, computer science, social sciences, psychology, multidisciplinary, and undefined. Furthermore, we limited our search to articles, articles in press, and conference papers, written in English only. As for ACM DL, we did not apply any restriction because it already focuses on computer science publications. These queries retrieved 3519 entries: 2825 in Scopus and 694 in ACM DL. We exported the results in a table, rearranged the columns with the publications details in order to make them uniform, and deleted the duplicates. Eventually, our initial tentative corpus had 3186 papers.

\section{B. Selection Criteria}

The corpus has been refined by checking the appropriateness of each paper to the following criteria.

1) The papers had to address a sport, in the strict sense of an intentional and specific physical activity conducted for its own sake. Wearables for fitness were included in this definition. However, we excluded all those wearables specifically aimed at promoting health goals (e.g., losing weight), as well as those explicitly designed for physical activity that could be conducted while performing other tasks, e.g., pedometers measuring the steps taken while going to work or shopping.

2) The papers had to present an interactive artifact, that is, a device providing an interface purposefully designed for being interacted with end users in the sports domain (e.g., athletes, coaches, and audience). With this criterion, we excluded all the works pertaining to human activity recognition, as this research field focuses on the devices' sensing capabilities, rather than on their "human aspects"; even though such works may provide data visualizations, these are not specifically designed for end users in the sports domain.

3) The papers had to present a study with end users. This criterion was included to ensure that the review pertained to the field of HCI.

The corpus was divided into four parts, and each part was assigned to one of the authors of this paper. Each author evaluated her/his assigned papers by reading the title, the keywords, and the abstract, thus discarding those not corresponding to the above criteria. This first selection produced a corpus of 112 papers. 
The great diminution from 3186 to 112 papers was due to the fact that the majority of them investigated activity recognition techniques rather than interactive artifacts. After this selection, the remaining papers have been carefully read by all the authors and discussed. This thorough reading allowed for the identification of those papers that did not fully satisfy the three selection criteria, which were thus discarded. Moreover, whenever the corpus presented more than one paper by the same authors and on the same project, we selected the most complete one for our analysis while discarding the others. Eventually, our corpus includes 57 papers. However, among the discarded papers, four were retained in a separate folder because they were considered inspirational and useful for understanding the topic [18]-[21]. These comply with at least two selection criteria.

\section{Analyze and Present}

The analysis was inductively oriented: once the corpus was defined, the authors read the papers to identify recurrent themes. As a first step, the open coding, each author separately assigned one or more conceptual labels to each paper. As for the second step, the axial coding, the concepts were grouped into coherent conceptual categories. Again, this was made by each author separately. Then, the authors discussed together the defined categories and reconciled them. Finally, the conceptual categories were connected one to the other in a coherent explanatory scheme. Five main themes emerged from the analysis: research perspectives and methodological approaches, types of sports and sportspeople, roles of wearable devices in sports, wearability, and type of feedback.

\section{FINDINGS}

\section{A. Research Perspectives and Methodological Approaches}

We recognized three different research perspectives in our corpus: the technology-driven, the design-driven, and the acceptance study perspective. The technology-driven perspective consists in the presentation and discussion of novel devices (usually prototypes) in a specific sports context. Papers adopting this perspective focus on the potentialities of a specific technological solution: the design idea is often presented in terms of requirements and the motivation for its development is commonly taken from the literature. These studies present user evaluations, albeit only preliminary, revolving around usability aspects. We identified 31 papers belonging to this perspective [22]-[52].

The design-driven perspective includes ten papers [53]-[62]. This perspective focuses on design aspects and often employs a constructivist stance, involving potential users in validating the proposed design idea. Two papers use a research-through-design approach [56], [60], while one employs design workshop activities [59]. One paper uses interviews with potential users [54], one adopts observation and semistructured interviews [55], and one employs content analysis of online reviews of wearable devices [57]. Others engage users through questionnaires or online surveys [53], [58], [61]. Some of these papers involve users for both gaining insights to be used in the design process, and assessing the designed prototype [53], [56], [58], [59], [61], [62].
TABLE I

RESEARCH METHODS EMPLOYED

\begin{tabular}{|c|c|c|c|}
\hline Perspective & $\begin{array}{l}\text { User Needs } \\
\text { Elicitation }\end{array}$ & $\begin{array}{l}\text { Evaluation } \\
(\mathrm{Lab})\end{array}$ & $\begin{array}{c}\text { Evaluation } \\
\text { (Field) }\end{array}$ \\
\hline Tech-driven & {$[22],[23],[38]$} & $\begin{array}{l}{[24],[28]-[33],} \\
{[36],[39]-[44],} \\
{[47],[48],[50]-} \\
{[52]}\end{array}$ & $\begin{array}{l}{[25]-[27],[34],} \\
{[35],[37],[45],} \\
{[46],[49]}\end{array}$ \\
\hline Design & $\begin{array}{l}{[53]-[55],} \\
{[57]-[62]}\end{array}$ & $\begin{array}{l}{[53],[56],[58],} \\
{[61],[62]}\end{array}$ & [59] \\
\hline Acceptance & $\begin{array}{l}\text { [64]-[71], [74], } \\
{[75],[78]}\end{array}$ & {$[63]$} & $\begin{array}{l}{[65],[72],[73],} \\
{[76]}\end{array}$ \\
\hline
\end{tabular}

The acceptance study perspective includes 16 papers. This perspective entails the conduction of (relatively) large user studies, investigating the acceptability and the adoption of specific wearable devices [63]-[78]. Seven works use in-depth interviews [64], [66], [68], [71], [72], [75], [78], four use online surveys [63], [67], [73], [77], and one uses questionnaires [76]. Two papers adopt a combination of methods: online surveys and interviews [69], or logs and online survey [65]. One paper employs sentiment analysis of customers' comments on Amazon.com [70]. Auto-ethnography is also employed by one work, as a pilot approach to prepare for a contextual analysis [74]. In all the cases, the studies are focused on commercial products.

A relevant aspect that is worth to be deepened relates to the research methods employed across the three perspectives. These can be divided into user needs elicitation studies, addressed to discover requirements to develop prototypes or define opportunities for creating novel devices, and evaluation studies, aimed at assessing novel or commercial solutions. Table I outlines how the studies are distributed among the three perspectives (some works present more than one study thus belonging to multiple categories).

As for the user needs elicitation, questionnaires (both paper and online based), interviews, and observation have been mostly employed. Some of the studies using questionnaires recruited a large number of participants, such as Gradl et al. [67], who involved 227 athletes, Stewart et al. [53], who collected 323 answers from roller derby skaters, and Havlucu et al. [69], who enrolled 1567 members of the Turkish Tennis Federation. However, the majority of them involved more limited samples, such as Aggravi et al. [22], who involved one skiing instructor and seven blind skiers, or Zhao et al. [23] who recruited five participants. Interviews conducted to identify user requirements commonly involved a small number of users (less than 20): For example, Carrington et al. [64] interviewed five wheelchair athletes and three therapists, while Pan et al. [54] interviewed seven weightlifters. Conversely, interviews investigating the situated use of commercial devices involved a higher number of participants (20 or more): Rapp and Tirabeni [75] interviewed 20 elite and amateur athletes to discover how wearables integrate into their sports activities; Fritz et al. [66] recruited 30 users of commercial activity trackers to gather insights on how to design wearables that can be used over long terms; Gui et al. [68] 
interviewed 31 WeRun users to investigate the influence of preexisting social network in sharing habits; and Jaharrai et al. [71] interviewed 29 people about their motivation to do sports. Observation and contextual interviews, instead, are used to a much lesser extent. Mencarini et al. [55] observed 6 indoor climbing lessons and interviewed 17 trainees and mountain guides, whereas Patel and O'Kane [74] observed 11 participants while exercising at the gym.

As for the evaluation, the methods employed can be divided into experimental lab studies and field studies, both using a variety of techniques to gather measures and insights.

Experimental lab studies were conducted in "controlled situations" often opting for quantitative inspection techniques or mixed methods. Bogers et al. [56], for instance, employed semistructured interviews, questionnaires, and log data analysis to evaluate jerseys measuring ball possession and giving feedback using light stripes. It is worth noticing that experiments rarely employed longitudinal designs, control groups or pre/post-test interventions. There are, though, some exceptions: For example, Bice et al. [63] designed an 8-week, pre-/post-test intervention experiment to assess the effects of wearables on physical activity motivation; Strohrmann et al. [44] employed a control group to evaluate how an IMU connected to a smartphone application may improve running technique; Zhao et al. [24] used a between-subject design with 36 participants providing pre/postquestionnaires.

Field studies, instead, were aimed at assessing wearables "in the wild" leaving users free to use them during their everyday sports and fitness practices. Fedosov et al. [25], for instance, monitored participants in a weeklong winter seminar to study the perceived usefulness and usability of a wearable augmented reality (AR) system for sharing content on ski resort maps. Mauriello et al. [26] used observation, interviews, and questionnaires to evaluate a wearable e-textile display aimed at supporting group running. A residual number of works exclusively asked for users' opinions using either questionnaires or semistructured interviews. Kreitzberg et al. [72], for instance, interviewed 25 participants to explore how and with whom people communicate fitness tracker messages. Kräuter et al. [27] employed a questionnaire to assess users' feelings toward a wearable prototype for cycling.

\section{B. Types of Sports and Sportspeople}

The types of sports and sportspeople addressed in our corpus vary considerably, even though regularities are present. Nine papers do not focus on a specific sport but rather consider sports in a very generic way [24], [28], [29], [57], [67], [73], [75]-[77]. Seven papers refer to fitness [23], [63], [66], [70]-[72], [74]. Six papers point to broad categories of sports such as lower limb sports [30], endurance sports [78], strategic sports [31], field sports [32], Olympic sports [58], or wheelchair sports [64]. The remaining ones address a wide range of different sports: eight are on skiing/snowboarding, seven are on running, and four papers focus on climbing. Sports such as cycling, tennis, swimming, and martial arts are only investigated in two papers each. Finally, other sports addressed (each one mentioned in one paper only) are diving, roller derby, rowing, badminton, and weightlifting.

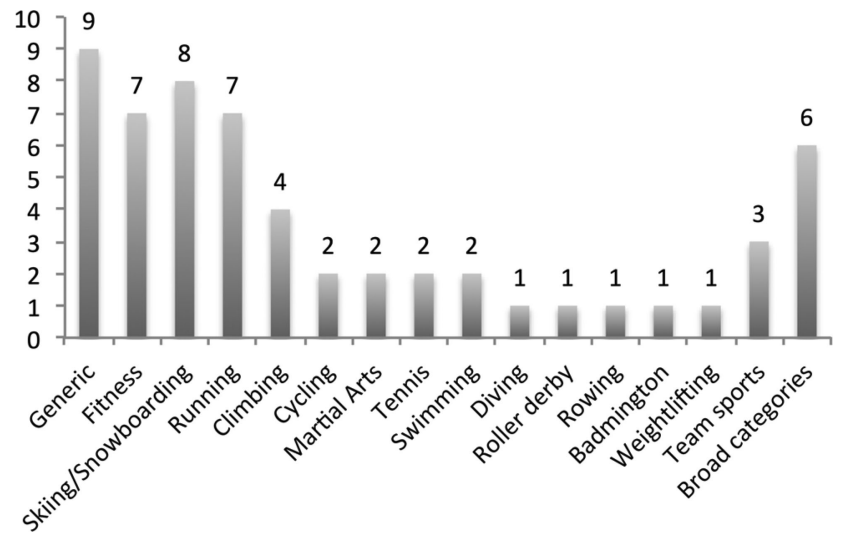

Fig. 1. Sports addressed in our survey.

Only three team sports are present in our corpus: basketball [56], football [33], and hockey [59]. No paper considers motor sports such as car or motorcycle racing. See Fig. 1 for an overview of the sports addressed in the corpus.

For what concerns the targeted users, most papers (27 out of 57) focus on generic sportspeople (e.g., without specifying their level of expertise, such as beginner or elite, or referring to a generic practitioner). Two papers address disabled sportspeople, without specifying their level of expertise [22], [34]. Five papers consider elite athletes [35], [36], [59], [60], [69] and two impaired elite athletes [37], [64]. Three papers consider both elite and amateur sportspeople [38], [75], [78], and three amateur sportspeople only [39]-[41]. Beginners are investigated in 11 papers, e.g., [42]-[44], [55], [61]. It is worth noticing that among the papers addressing beginners, three are focused on climbing (out of a total of four papers addressing climbing). Probably, this is due to the fact that some sports (e.g., climbing) need a higher technical expertise to be practiced than other sports (e.g., running), thus requiring research on technology capable of supporting novice athletes. Four papers do not investigate sportspeople directly, but target audience of sports events [29], [33], [58], [65].

Other sports actors, such as coaches, teammates, judges, therapists, and social network audience, are also mentioned in our corpus (19 papers out of 57), highlighting the social dimension of sports. Health professionals occur twice [64], [59]. Coaches are considered in eight papers [22], [32], [34], [36], [45], [46], [55], [60], sports partners, and teammates are investigated five times [25]-[27], [31], [56], whereas judges are mentioned only in [35]. Finally, social network audience is considered in three works [68], [72], [77].

\section{Role of Wearable Devices in Sports}

We identified three nonexclusive roles that wearables may play in the sports practice, namely, enabling, improving, and augmenting one or more sport-related aspects. Enabling means that wearables allow users to do something that would be impossible to do without technology; improving means that wearables are meant to support the user in doing something in a better way; augmenting relates to do something in a different way than how it was done without technology. 
TABLE II

ROLE OF WEARABLE DEVICES IN SPORTS

\begin{tabular}{|l|c|l|l|}
\hline $\begin{array}{c}\text { SPORTS } \\
\text { ASPECT }\end{array}$ & \multicolumn{1}{|c|}{ ENABLING } & \multicolumn{1}{|c|}{ IMPROVING } & AUGMENTING \\
\hline \multirow{3}{*}{$\begin{array}{l}\text { Physical } \\
{[22],[32],[34],}\end{array}$} & $\begin{array}{l}{[28],[30],[35],[39]-} \\
{[45],[43],[51],[52],} \\
{[45],[47],[48],}\end{array}$ & $\begin{array}{l}{[53],[54],[66],[75],} \\
{[78]}\end{array}$ & {$[50]$} \\
\hline $\begin{array}{l}\text { Cognitive- } \\
\text { emotional }\end{array}$ & & $\begin{array}{l}{[26],[36],[38],[59],} \\
{[60],[62],[63],[69],} \\
{[71],[73],[74]}\end{array}$ & $\begin{array}{l}{[23],[24],[49],} \\
{[56],[67]}\end{array}$ \\
\hline Social & {$[33],[58],[65]$} & $\begin{array}{l}{[25],[27],[68],[72],} \\
{[77]}\end{array}$ & {$[29],[31],[46]$} \\
\hline
\end{tabular}

In order to analyze the kind of support given by technology, we distinguished three aspects of the sports practice, namely the physical, the cognitive-emotional, and the social aspects.

1) Physical: It relates to performance and motor skills acquisition for specific sports techniques; while the former is generally a concern for experienced athletes, the latter pertains mainly to beginners. In sports, a good technique means how well a skill is performed, whereby a skill is an achieved ability to bring about predetermined results, with a maximum of certainty and often with the minimum of outlay time, energy, or both [70], for example, the correct use of feet in climbing.

2) Cognitive-emotional: It comprises emotions, flow, mental control, and other psychological factors such as motivation, i.e., the promotion of the desire or willingness to do or achieve something. Motivation is important in sport: It can be defined as the direction (i.e., the situations, practices, etc., to which an athlete is attracted) and intensity (i.e., how hard she tries) of one's effort, whereby the temporal dimension is relevant, i.e., how sustained or lasting that effort is [79].

3) Social: It encompasses the broad dimension of social relations and inclusiveness; it refers to activities that allow people to meet each other for pleasure. It also concerns interpersonal and organizational communication, both verbal and nonverbal [80].

As summarized in Table II, the main role emerging from our corpus is to improve a specific sports aspect (30 papers), while few papers aim to enable (15) or augment (9) a sport. It is worth noticing that some papers could be classified into multiple categories as they address more than one aspect of sports: however, for the sake of clarity, we considered only the main role, i.e., the role that is more extensively discussed in the paper. Three papers were not classified with reference to the role of wearable [57], [70], [76]. Moreover, we could not identify any example in which technology was meant to enable the cognitive-emotional aspect.

As for the enabling role, 15 papers either support the acquisition of motor skills by beginners or allow impaired individuals to practice a sport. Franke and Lukowicz [61] provide rowing beginners with a system that allows to both train and monitor their technique without requiring the direct supervision of an instructor. Feeken et al. [43] design a wearable system that facilitates the development of technical skills of beginner climbers. Chen et al. [42] develop a virtual coach that helps marathon beginners adjust their speed based on their heart rate so that their body can react in time to maintain the required training intensity. Three papers focus on enabling the social experience [58], [65], [33]: They present tools for making the enthusiasm of remote audience visible in order to enable the public's participation during sports events.

The improving role is the most frequently discussed role in our corpus, being present in 30 papers. A total of 14 papers focus on improving the physical aspect of sports. For example, Ladha et al. [39] propose a performance analysis system that replicates expert assessments, thus representing a first step toward an automatic coaching system for climbing. Hassan et al. [47], Seuter et al. [28], and Strohrmann et al. [44] propose systems based on different types of feedback to improve the performance in run training. Chi et al. [35] aim to better the judges' performance assessment, giving help in evaluating sparring matches in martial arts. Rapp and Tirabeni [75] explore how wearables impact on the athlete's performance, finding that their effect may change depending on her level of experience. A total of 11 papers focus on the improvement of the cognitive-emotional aspects of sports. Kidman et al. [36], for instance, aim to ameliorate the athletes' self-awareness of both short- and long-term fatigue in order to control task execution consciously. Jarrahi et al. [71] investigate the impact that wearables have in motivating people to do physical activity. Havlucu et al. [60], [69] highlight the importance of flow in the sports performance of tennis players and propose concepts that help to maintain it. Five papers focus on aspects related to the social experience: For example, Kraeuter et al. [27] propose a system using gesture recognition and a series of light emitting diodes (LEDs) sewed on the back of cyclists' shirt in order to help them communicate when they are on the road, thus improving interpersonal communication. Gui et al. [68] and Stragier et al. [77] investigate the impact of sharing information about sports activity on social networks.

The augmenting role is the less discussed role in our corpus. This group includes nine works introducing interactive elements into the sports activity so that the nature of the sport itself is modified (e.g., in its rules and objectives). Three papers focus on augmenting the social experience. Tomitsch et al. [29] use wireless wearable motion sensors to augment spectator participation in loco, changing their opportunities for intervening. Weilenmann et al. [46] and Kono et al. [31] present wearable systems for augmenting the social experience of groups of collocated sportspeople. The augmentation of the physical aspects of sports is addressed only by Niforatos et al. [50], who developed a system for augmenting the skiers' peripheral view, allowing them to perceive whether somebody is getting to their back. Five papers present devices that aim to augment cognitive-emotional aspects: For example, Colley et al. [49] investigate the effects of presenting on a head-mounted display (HMD) an alternative virtual reality (VR) view while the skier is on a real slope, creating a blended virtual/real experience, whereas Gradl et al. [67] explore the acceptance of VR and AR systems among athletes.

\section{Wearability}

The term wearability refers to the ability of an artifact to be worn on the human body [2]. It is worth noticing that a good 




Fig. 2. Body locations for sensors and actuators addressed in our survey.

wearability is not only a matter of physical comfort, but also of social and psychological comfort [81], in order to be effective in conveying information and accessible for interaction [82].

Few articles (8 out of 57) in our corpus directly address the topic of wearability [48], [55], [57], [59]-[62], [64]. These are exploratory papers presenting investigations that may inform the design of new wearable devices. Even though the term "wearability" is not explicitly mentioned, these authors attempt to find the best body location to place the device in order to ensure comfort, accuracy of sensing, and perceptibility of feedback. Bächlin et al. [48] and Kosmalla et al. [62], for instance, attempt to discover the best form factors, body locations, or feedback modalities in swimming and climbing, respectively.

The majority of the selected papers (29 out of 57) present new wearable prototypes or adapt multipurpose commercial wearables to the sports domain (e.g., goggles) without discussing their wearability. Yet, by analyzing the proposed devices, they offer implicit motivations for their design choices. The most common form and location are a band or a watch to be placed on the wrist (21 papers). This is convenient because it exploits the conventional location of watches and bracelets, which are already culturally recognized and accepted. Furthermore, placing the device on the wrist allows feedback to be visualized easily, and facilitates the collection of both physical and physiological data, e.g., heart signal and body movements. Other body locations explored in the corpus are the head [25], [31], [38], [45], [49], [67], the neck [58], the thorax [26], [32], [36], [42], [56], the arms [34], [44], [65], the legs [30], [37], the feet [43], [47], and multiple locations all over the body (9). This last group includes wearable systems composed of different devices for sensing and sending feedback [27], [28], [52], [53], [65], as well as wearable systems for full-body sports [35], [40], [41], [54]. See Fig. 2 for an overview of the body locations addressed in the corpus.

By analyzing the papers that have moved beyond the "watch metaphor," it emerges that the decisions on the shape to be given to the artifact, as well as on the position of the body where to place it, are made on the basis of two reasons: 1) the role of the device (e.g., augmenting the experience, improving the communication with teammates, etc.); 2) the possibility of embedding the device in an artifact already used during the sports practice (e.g., clothes or gear), or the need to create a new "object." It is also apparent that the studies aiming to augment the sports experience typically rely on HMDs, using AR or VR. HMDs have been studied both in sports where goggles are already part of the athlete's equipment, such as skiing and snowboarding [25], [31], [49], [50], and in sports where the enhancement of the experience would require an additional artifact [38], [45], [67].

By contrast, research aimed at supporting the athlete's performance during training or learning defines the wearable's shape and location on the basis of the body parts involved in the sport (for ensuring a more precise movement detection), as well as the target of the feedback (for a better feedback perceptibility). To ensure optimal sensing, they can be placed on the athlete's feet [41], [43], [44], legs [28], [30], chest [42], or wrist [39], depending on the types of movements required by each sport. To ensure the perceptibility of the feedback, wearables are typically positioned on the athlete's arms [44], [65], ears [37], or waist [36]. When the feedback targets other athletes involved in the sports activity (i.e., teammates or opponents), it is commonly embedded in the athlete's apparel and displayed on the thorax to be more visible [26], [27], [56]; instead, when it is addressed to external actors, such as judges and audience, the information is captured by sensors placed on the athlete and is displayed on screens [29], [35], [58].

\section{E. Type of Feedback}

The last theme concerns the type of feedback provided. Athletes rely either on explicit indicators, such as the achievement of the pursued goal, or on feedback responses, which can be inherent or augmented [83], in order to assess their performance. Inherent feedback (also called intrinsic) is the information originating from the athlete's perception of her own movements and position in space (i.e., proprioception), while augmented feedback (or extrinsic) is the information coming from an external agent [83], such as the coach or video-based motion analysis [84].

Augmented feedback is fundamental for learning and improving because it helps athletes categorize their internal sensations and better understand the mechanisms underlying their performance: this is the kind of feedback that wearables can provide. Augmented feedback (from now on called just "feedback") can be categorized according to the timing of delivery. It is defined as concurrent if delivered while executing the movement (i.e., in real time), and terminal if it is given once the movement is concluded [85]. The decision about whether using concurrent or terminal feedback depends on the kind of task required by the sport as well as on the content that the feedback aims to express. Indeed, feedback can express either knowledge of performance, when it refers to the quality of movements, or knowledge of results, when it refers to the goal/level achieved [85]. We identified 37 papers that present wearables providing concurrent feedback, eight papers presenting wearables giving terminal feedback [23], [24], [33], [39], [59], [68], [71], [77], and six papers that discuss wearable systems providing both (mixed feedback) [63], [66], [72]-[74], [78]. Six papers do not directly address the topic of feedback [45], [57], [64], [69], [70], [75], [76].

Another distinction can be made according to the modality used to convey the feedback (see Fig. 3). The most used modality across all the types of delivery (i.e., concurrent, terminal, and 


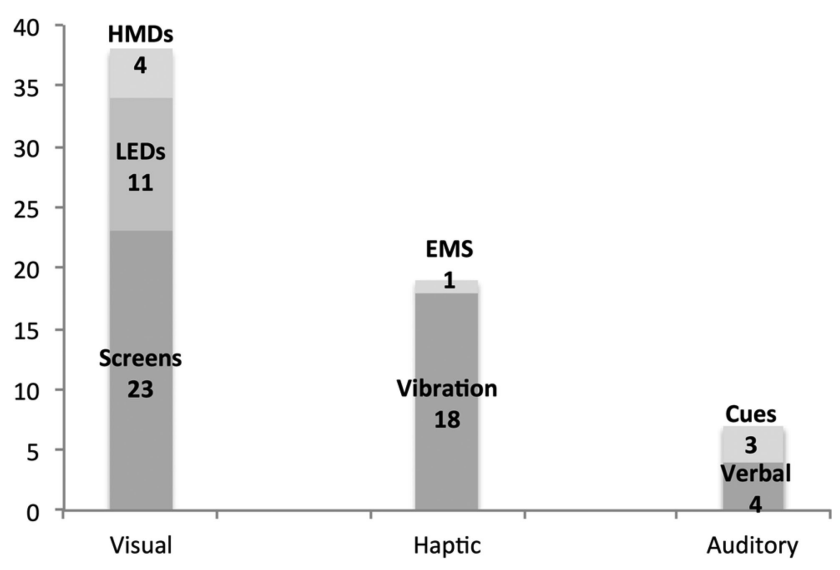

Fig. 3. Feedback modalities and types of delivery addressed in our survey.

mixed) is the visual one, with information prompted on screens (23 wearable systems), LEDs (11), or HMDs (4).

In particular, the devices adopting terminal and mixed feedback rely on visual modality only. Terminal feedback is provided through mobile apps [23], [24], [33], [59], [68] or websites [39], [71], [77]. Likewise, mixed feedback is usually prompted in real time on the wearable display in the form of a "summary" of the athlete's progress, and then visualized on larger screens through more detailed representations using mobile apps or websites [63], [72]-[74]. The latter visualization allows also for sharing performance results on social networks, as discussed by Fritz et al. [66].

Concurrent feedback is delivered through three main modalities, visual, auditory, and haptic, depending on its target and purpose. Touch, using vibrations or electrical muscle stimulation, and hearing, through audible cues (e.g., beep or tone) or verbal instructions, are preferred when the feedback is directed at the sportsperson and the activity she is performing relies on her visual attention, e.g., [22], [30], [37], [40], [42], [53], [55], [60], [61]. In some of these cases [22], [41], [42], [55], [61], the haptic or auditory information enables the coach to send a feedforward rather than a feedback, i.e., an instruction about what to do preceding the action rather than following it. In case of multiple feedbacks conveyed to different stakeholders at the same time, the visual channel is dedicated to the audience [65], the coach [36], the teammates [26], [27], [56], or the judges [35], while the athletes are informed through haptic and/or audio stimuli. Visual feedback is used to inform directly the sportsperson when her improvements depend on the visual feedback itself, e.g., when the feedback mirrors her performance [28], or when it is aimed at augmenting her performance [25], [31], [38], [45], [49], [67]. The choice about feedback modality also depends on the kind of sport practiced: explorative studies that compared the three different modalities found that visual feedback would work better for swimmers [48], whereas sound and vibration would work better for climbers [62].

\section{DISCUSSION}

The analysis presented above depicts an area of HCI that is still in its early stages, being driven more by technological advancements than by "human goals and needs," which traditionally orient HCI research. In the following, we discuss six directions for a possible roadmap of research in this area.

\section{A. Explore Different Form Factors and Types of Feedback}

The majority of wearable devices we analyzed are designed to be worn on the wrist and are similar in shape to watches and bracelets. The watch form is convenient because it leverages a widespread and cross-cultural convention about where to wear an "artifact" in order to have access to some kind of information. Yet, HCI research could push the wearables' product design forward by investigating new form factors and body locations. Through a thorough analysis of the sports type, i.e., the movements involved in a certain sports practice, as well as the gear and the apparel used in it, the role that the wearable may fulfill, and its function (sensing, sending feedback, or both), it would be possible to envision new form factors and interaction modalities. By and large, HCI research should aim to map the dimensions involved in the design of wearables for sports (sports type, role, and function).

The analysis also makes apparent that the feedback delivered by current wearables is mostly concurrent [78]. Although this makes sense in several situations and for many sports, terminal feedback is definitely worth to be explored. The integration of concurrent and terminal feedback, and how we can differentiate their use depending on the athlete's situations and goals, should be investigated as well. This would open new opportunities for studying the expressive abilities of feedback both in real time (i.e., how much information is possible to deliver in real time, as well as is perceivable and understandable by sportspeople while engaged in their performances) and after the experience (when there is time for reflection on the experience just lived).

\section{B. Move Beyond "Generic" "Individual" Athletes}

At present, HCI research seems to focus on individual sports and on generic sportspeople (that is, without specifying their level of experience, ability, or frequency in training). Most wearable devices are designed for individual sportspeople/athletes (only three team sports are present in our corpus). Likewise, evaluations usually involved athletes as individuals and not as a group/team. Indeed, there are few examples considering the social dimension of sports. For example, Chi et al. [35] focus on pairs of martial arts athletes, studying piezoelectric force sensors that detect when a significant impact has been delivered to a competitor's body; Kräuter $e t$ al. [27] recruit a regularly meeting group of cyclists to evaluate a prototype that enhances group communication; and Mauriello et al. [26] evaluate light jerseys with a small team of basketball players. Still, there are many possibilities to widen the "unit" engaged in the interaction with the device.

On the one hand, focusing on team sports may open completely new design questions and opportunities, e.g., for improving the communication among the athletes and with the coaches. On the other hand, considering collaborative behavior at the wearable device layer, with particular reference to multiuser task/activity applications, may set new directions for wearables for sports research. For instance, wearables may become a collective support for strategy definition in team sports, 
whereby athletes may cooperate together by using the devices in order to tune their ongoing performance and refine their goals in real time. Likewise, the wearable may "mediate" the relationship between the athlete and the coach, helping them collectively define the workouts, or develop a "shared knowledge" that may benefit the athlete's understanding of her own body. In sum, there are a variety of collaborative activities in the sports domain that could be supported by wearables by enabling multiple users to work together on the same task.

A further research direction is to consider the type of sportspeople involved in the interaction with the device. Few studies focus on elite sportspeople or beginners, rather preferring to target "average" athletes. Technology may support athletes in different ways along the spectrum of their expertise and sports knowledge. Actually, Rapp and Tirabeni [75] highlighted that athletes at different levels of experience have different information needs and engage in different sports practices. This should be further explored in future studies. Similarly, few papers focus on designing for disabled athletes. Moreover, they consider physical impairments, while ignoring cognitive ones. A research opportunity for using wearable technology in sports could be to enable cognitively impaired individuals to recognize and manage emotions or cognitive states relevant to certain sports activities, e.g., by supporting their memory functioning, focusing their attention, or reducing their emotional distress (e.g., in autistic individuals).

\section{Push the Boundaries}

Regarding the roles that wearables could play in sports, our review identified two main gaps in the current literature (see Table II): it seems that there is no research on wearables enabling the cognitive and emotional aspects of sport, as well as on their use for augmenting physical skills (with the exception of [50]). It is apparent, instead, that the majority of works we analyzed have produced and investigated wearables for enabling or improving physical aspects of sports. This may be due to the relative ease of implementing measurement of performance in many popular sports, like cycling and running, as it depends on variables that can be effortlessly collected by the sensors embedded in the device (e.g., speed). Nevertheless, the sports experience cannot be reduced to the mere physical effort and performance as it entails a cognitive/emotional dimension that is harder to measure but interesting to explore.

Cognitive and emotional aspects are important in the sports practice to support motivation and commitment [86] as well as to prevent burnout [87] and facilitate coping strategies [88]. With few exceptions (i.e., [62] and [75]), cognitive aspects related to performance have not received much attention in our corpus. Interesting possibilities for research and design can thus be opened by the exploration of psychological, emotional, and behavioral variables, such as self-efficacy and motivation. For example, appropriate visualizations may support athletes in reflecting on their own expectations and level of stress, as well as provide coaches with tools to foster athletes' motivation while preserving their safety. By considering the athlete's lived-experience, its monitoring, and integration into reflective practices on the ongoing performance, Hirose et al. [19] proposed a system that records one's third-person view while diving, providing a sort of "enhanced eye" that augments the sports experience if used in real time.

Augmenting the athlete's physical abilities is another interesting line of research for HCI. As wearables are strictly connected to the human body, technology might empower athletes by giving them more strength, speed, or ability to deal with fatigue, as well as by expanding the kinds of movements they are able to enact. The lack of research on this topic may be due to the intrinsic assumption that the body and its development must remain "untouched" by agents external to the sports domain: the body in sport must be improved through training and sacrifices, whereby any external aid could appear to violate this assumption, falling within the "cheating" category. Although augmentation may evolve toward the idea of enhancement (see, e.g., [89]), be perceived in contradiction to sports values (see, e.g., [55]), and conceptually close to doping, it could also open interesting perspectives when applied to accessibility issues. For example, e-bikes have been proven to widen the accessibility of biking (e.g., [90]): a positive attitude toward technology for augmenting "the human" can pave the way for further investigations in this domain.

In this perspective, many research questions related to wearables and sport have not yet been addressed by HCI researchers. For example, are wearable excessively "quantifying" the sports experience turning something physical into digital data that can be rationally examined? How, and to what extent, do wearables produce psychological effects on athletes? Are wearables changing the organizational practices in the sports domain, reconfiguring roles, and modifying rules?

\section{Explore the Evolution of Sports}

Surprisingly, there is currently a lack of disruptive ideas about how technology could change the sports domain itself, by giving life to new kinds of sports, or creating novel opportunities for athletes. Despite the sci-fi imaginary depicting future societies where team and individual sports are evolved thanks to novel enabling technologies (for example, in movies like "Rollerball" and "Futuresport"), there is no research on how wearable technology may change the rules of a sport. Actually, an interesting example that goes along this line of research is represented by Hayakawa et al. [18]. Even though it has not been included in our corpus as it lacks a proper user study, it discusses a new sports genre, the "Aerial Sports," whereby a wearable HMD integrates a camera view from a flight drone unit with the user's voluntary motion. This supposedly creates a new sports experience in which the athlete enjoys crossing her physical limitations, such as height and gravity, by diving into the drone and experiencing the binocular stereoscopic sensation of flying while using her limbs effectively. In this respect, interesting insights may come from initiatives like the symposium on "superhuman sports" [91] organized under the auspices of ACM.

\section{E. Interconnect Different Systems}

Technical discussion about interoperability among different standards and communication technologies is missing in our corpus. This comes as no surprise as HCI works rarely tackle 
technical issues directly. However, much more surprising is the complete absence of insights on the possibility of integrating devices at the application level, allowing designers to design wearable systems exploiting multiple platforms in homogeneous or cross domains, as well as users to exchange data across different services within a unique user experience. Actually, the wearables we analyzed are thought as stand-alone systems, whereby their integration into a wider ecosystem is not taken into account.

There are instead many opportunities coming from the integration of wearables with Internet of Things (IoT) via cloud computing and/or edge infrastructures. Aloi et al. [92] highlight how interoperability could allow the integration of different data sources, favor the distribution of data on the best device available, enable the interaction between heterogeneous devices, and give life to pervasive dynamic services. These opportunities could be relevant for the sports domain as well and need further research. For instance, interoperability could allow to design wearable technologies for motor sports (which are currently absent in our corpus) which may coordinate with other technological instruments (such as a car or a motorbike). Further, it may increase the kind of data considered by the wearable, which are now siloed and circumscribed to the sport practiced, by connecting them with information coming from other IoT devices (e.g., medical wearables), extending the parameters monitored to multiple aspects of the athlete's life. Moreover, interoperability may increase the opportunities for creating dynamic visualizations displaying concurrent and terminal feedback on the best screen available (also leveraging the resources embedded in the "environment").

\section{F. Improve Methodological Rigor}

By looking at the methods employed across all the three identified research perspectives, most of the user studies lack thoroughness, rigor, and are not fully capable of providing in-depth insights on the "human" impact of wearables.

By and large, most of the qualitative studies examined show very poor description of the methods employed and surprisingly present an incomplete recounting of the results. For example, the studies focused on requirement gathering often lack appropriate observational and ethnographic research. "Full" ethnographies implying a long period of observation and/or participation into sports activities are absent in our corpus, signaling that at present the HCI community has limited observational data on how wearables are, or can be, integrated into the sports practices. Likewise, in-the-wild evaluation studies are currently needed. Schlögl et al. [21] highlight that there is still a number of key problems requiring real-life user studies to be solved in order to gain a holistic understanding of the challenges that arise from interacting with wearable technology. Some of the studies claiming to be "in the field" deployed their systems in an ecological setting, but not in completely "natural" situations: For example, they introduced artificial tasks created by the researcher [25] or lacked rigor in explaining the study setting and the sample involved. Further, evaluations have been mainly conducted in the short term through a limited number of trials or during a limited number of days. Of course, acceptance studies are complicated by the fact that extended use might be difficult to assess with research prototypes. Indeed, all the papers in the "acceptance study" category investigate the impact of commercial wearables.

The quantitative studies seem to raise the same concerns: most of them were explorative and involved a limited number of participants, with rather weak outcomes in terms of research design and statistical validity. We did not find randomized control trials among the examined papers, while questionnaires present little statistical elaboration and poor description of the used methods. Long-term impacts on psychological and behavioral variables and eventual side effects of wearable use are not considered as well.

Given this, we believe that the field needs more experimental studies reporting effect size, employing longitudinal designs and involving control groups, as well as comparing different designs, with reference to the wearables' shape, wearability, functionalities, and visualizations. As for the qualitative research, researchers should focus on how wearables are used in sports practices, aiming to understand their experiential effects, the "how" and "why" they support or not the athletes" situated goals. As Rapp and Tirabeni noted, HCI research on wearable and sports lacks reports on how wearables are subjectively "lived" [75], and how they are appropriated by athletes for their own situated purposes, even against the designer's intentions. Contextual interviews (like those reported in [75]), diaries, and ethnographies exploring how wearable devices affect the athlete's experience, as well as how contextual factors and athletes' idiosyncrasies may influence wearables' acceptability and efficacy, should be conducted in the next years.

\section{CONCLUSION AND Future WORK}

In this paper, we presented a literature review following the grounded theory literature review approach [15]. We analyzed 57 papers published in scientific conferences and journals from 1999 to 2018 with the aim of understanding how current HCI research tackles wearable technology in the sports domain. Our analysis shows that HCI research in this field is, in many respects, in its infancy: Despite its allegedly interest in the "human aspects" of interaction, here HCI is still driven by technical aspects of design, while overlooking the impact of technology on the user experience.

We identified six directions for moving the HCI research on wearables for sports forward: 1) While current research is dominated by the metaphor of the wrist watch and by delivering concurrent feedback, times might be ripe to map the dimensions involved in the design of wearable for sports (role, function, and sports type) to the types of feedback that can be provided; 2) we believe that research should move beyond proposing devices addressed to "average" "individual" athletes by targeting different sportspeople and exploring collaborative tasks; 3) future research should also consider the complex constellation of cognitive, emotional, and social aspects of the sports experience; 4) another intriguing line of research could be to explore how technological artifacts may change the sports experience by enabling radical new practices; 5) likewise, interconnecting 
wearable devices to the wider IoT ecosystem may enable new services and interaction modalities; and 6) finally, more rigorous methodological approaches are needed both for the analysis of the users' needs and for the evaluation of the technological artifacts developed.

There are many opportunities for future work. While at this stage we preferred to circumscribe our corpus to the sports domain for better pointing out its key issues and opportunities, $\mathrm{HCI}$ would benefit from widening the coverage of this review to other distinct, but related areas in the future. For instance, even though designed for different goals, contexts, and practices (i.e., health), wearable devices for physical rehabilitation are tightly related to sports activities. Singh et al. [93], for example, developed a wearable system that senses and sonifies movements supporting physical rehabilitation and pain management. While Vasconcelos et al. [94] proposed three exercise-agnostic games controlled by a wearable device, which can be used for a multitude of rehabilitation scenarios. These and other works in this vein could introduce new themes and give a supplementary perspective on how wearables are used while doing physical activity.

\section{REFERENCES}

[1] "The Future of sport 2.0," 2016. [Online]. Available: Futureof.org

[2] F. Gemperle, C. Kasabach, J. Stivoric, M. Bauer, and R. Martin, "Design for wearability," in Proc. 2nd Int. Symp. Wearable Comput., 1998, pp. 116122.

[3] G. Fortino, R. Giannantonio, R. Gravina, P. Kurylosky, and R. Jafari, "Enabling effective programming and flexible management of efficient body sensor network applications," IEEE Trans. Hum.-Mach. Syst., vol. 43, no. 1, pp. 115-133, Jan. 2013.

[4] O. D. Lara and M. A. Labrador, "A survey on human activity recognition using wearable sensors," IEEE Commun. Surveys Tut., vol. 15, no. 3, pp. 1192-1209, Third Quarter, 2013.

[5] Z. Wang, M. Jiang, Y. Hu, and H. Li, "An incremental learning method based on probabilistic neural networks and adjustable fuzzy clustering for human activity recognition by using wearable sensors," IEEE Trans. Inf. Technol. Biomed., vol. 16, no. 4, pp. 691-699, Jul. 2012.

[6] Z. Wang, H. Zhao, S. Qiu, and Q. Gao, "Stance phase detection for ZUPTaided foot-mounted pedestrian navigation system," IEEE/ASME Trans. Mechatron., vol. 20, no. 6, pp. 3170-3181, Dec. 2018.

[7] Z. Wang et al., "Inertial sensor-based analysis of equestrian sports between beginner and professional riders under different horse gaits," IEEE Trans. Instrum. Meas., vol. 67, no. 11, pp. 2692-2704, Nov. 2018.

[8] Z. Wang, M. Guo, and C. Zhao, "Badminton stroke recognition based on body sensor networks," IEEE Trans. Hum.-Mach. Syst., vol. 46, no. 5 , pp. 769-775, Oct. 2016.

[9] J. C. Maglott, J. Xu, and P. B. Shull, "Differences in arm motion timing characteristics for basketball free throw and jump shooting via a body-worn sensorized sleeve," in Proc. IEEE 14th Int. Conf. Wearable Implantable Body Sensor Netw., 2017, pp. 31-34.

[10] Z. Wang, J. Wang, H. Zhao, N. Yang, and G. Fortino, "CanoeSense: Monitoring canoe sprint motion using wearable sensors," in Proc. IEEE Int. Conf. Syst., Man, Cybern., Budapest, 2016, pp. 644-649.

[11] J. Pansiot, B. P. L. Lo, and G. Z. Yang, "Swimming stroke kinematic analysis with BSN," in Proc. Int. Conf. Body Sensor Netw., Singapore, 2010, pp. 153-158.

[12] S. Nylander, J. Tholander, F. Floyd Müller, and J. Marshall, "HCI and sports," Interactions, vol. 22, pp. 30-31, 2015.

[13] J. Webster and R. T. Watson, "Analyzing the past to prepare for the future: Writing a literature review," MIS Quart., vol. 26, pp. 13-23, 2002.

[14] F. Nunes, N. Verdezoto, G. Fitzpatrick, M. Kyng, E. Grönvall, and C. Storni, "Self-care technologies in HCI: Trends, tensions, and opportunities," ACM Trans. Comput.-Hum. Interact., vol. 22, no. 6, 2015, Art. no. 33

[15] J. F. Wolfswinkel, E. Furtmueller, and C. P. Wilderom, "Using grounded theory as a method for rigorously reviewing literature," Eur. J. Inf. Syst., vol. 22 , no. 1, pp. 45-55, 2013.
[16] B. G. Glaser and A. L. Strauss, Discovery of Grounded Theory: Strategies for Qualitative Research. Evanston, IL, USA: Routledge, 2017.

[17] K. Charmaz and L. L. Belgrave, "Grounded theory," The Blackwell Encyclopaedia of Sociology. Hoboken, NJ, USA: Wiley, 2007.

[18] H. Hayakawa, C. L. Fernando, M. H. D. Saraiji, K. Minamizawa, and S. Tachi, "Telexistence drone: Design of a flight telexistence system for immersive aerial sports experience," in Proc. 6th Augmented Human Int. Conf., 2015, pp. 171-172.

[19] M. Hirose, Y. Sugiura, K. Minamizawa, and M. Inama, "PukuPuCam: A recording system from third-person view in scuba diving," in Proc. 6th Augmented Hum. Int. Conf., 2015, pp. 161-162.

[20] K. Kunze, K. Minamizawa, S. Lukosch, M. Inami, and J. Rekimoto, "Superhuman sports: Applying human augmentation to physical exercise," IEEE Pervasive Comput., vol. 16, no. 2, pp. 14-17, Apr.-Jun. 2017

[21] S. Schlögl, J. Buricic, and M. Pycha, "Wearables in the wild: Advocating real-life user studies," in Proc. 17th Int. Conf. Hum.-Comput. Interact. Mobile Devices Services Adjunct, 2015, pp. 966-969.

[22] M. Aggravi, G. Salvietti, and D. Prattichizzo, "Haptic assistive bracelets for blind skier guidance," in Proc. 7th Augmented Human Int. Conf., 2016, Paper 25.

[23] Z. Zhao, S. A. Etemad, and A. Arya, "Gamification of exercise and fitness using wearable activity trackers," in Proc. 10th Int. Symp. Comput. Sci. Sports, 2016, pp. 233-240.

[24] Z. Zhao, S. A. Etemad, A. Whitehead, and A. Arya, "Motivational impacts and sustainability analysis of a wearable-based gamified exercise and fitness system," in Proc. Аnпu. Symp. Comput.-Hum. Interact. Play Companion Extended Abstr., 2016, pp. 359-365.

[25] A. Fedosov, E. Niforatos, I. Elhart, T. Schneider, D. Anisimov, and M. Langheinrich, "Design and evaluation of a wearable AR system for sharing personalized content on ski resort maps," in Proc. 15th Int. Conf. Mobile Ubiquitous Multimedia, 2016, pp. 141-152.

[26] M. Mauriello, M. Gubbels, and J. E. Froehlich, "Social fabric fitness: The design and evaluation of wearable E-textile displays to support group running," in Proc. SIGCHI Conf. Hum. Factors Comput. Syst., 2014, pp. 2833 2842

[27] N. Kräuter, S. Lösing, G. Bauer, L. Schwering, and M. Seuter, "Supporting safety in cycling groups using LED-augmented gestures," in Proc. ACM Int. Joint Conf. Pervasive Ubiquitous Comput., Adjunct, 2016, pp. 889892.

[28] M. Seuter, L. Opitz, G. Bauer, and D. Hochmann, "Live-feedback from the IMUs: Animated 3D visualization for everyday-exercising," in Proc. ACM Int. Joint Conf. Pervasive Ubiquitous Comput., Adjunct, 2016, pp. 904 907.

[29] M. Tomitsch, W. Aigner, and T. Grechenig, "A concept to support seamless spectator participation in sports events based on wearable motion sensors," in Proc. 2nd Int. Conf. Pervasive Comput. Appl., 2007, pp. 209-214.

[30] M. Fiorentino, A. Uva, M. Foglia, and V. Bevilacqua, "Asymmetry measurement for vibroactive correction in lower limbs mobility," Comput. Sci. Inf. Syst., vol. 10, no. 3, pp. 1387-1406, 2013.

[31] M. Kono, T. Miyaki, and J. Rekimoto, "JackIn airsoft: localization and view sharing for strategic sports," in Proc. 23rd ACM Symp. Virtual Reality Softw. Technol., 2017, Paper 3.

[32] R. Shiraishi, K. Sato, Y. Sano, and M. Otsuki, "Haptic directional instruction system for sports," in Proc. Int. AsiaHaptics Conf., 2016, pp. 361-368.

[33] M. Cruz, T. Romão, P. Centieiro, and A. E. Dias, "Exploring the use of second screen devices during live sports broadcasts to promote social interaction," in Proc. Int. Conf. Adv. Comput. Entertainment, 2017, pp. 318 338.

[34] J. Haladjan, M. Reif, and B. Brügge, "VIHapp: A wearable system to support blind skiing," in Proc. ACM Int. Joint Conf. Pervasive Ubiquitous Comput./ACM Int. Symp Wearable Comput., 2017, pp. 1033-1037.

[35] E. H. Chi, J. Song, and G. Corbin, “Killer App' of wearable computing: Wireless force sensing body protectors for martial arts," in Proc. 17th Annu. ACM Symp. User Interface Softw. Technol., 2004, pp. 277-285.

[36] E. M. Kidman, M. J. A. D'Souza, and S. P. N. Singh, "A wearable device with inertial motion tracking and vibro-tactile feedback for aesthetic sport athletes: Diving coach monitor," in Proc. 10th Int. Conf. Signal Commun. Syst., 2016, pp. 1-6.

[37] A. Muehlbradt, V. Koushik, and S. K. Kane, "Goby: A wearable swimming aid for blind athletes," in Proc. 19th Int. ACM SIGACCESS Conf. Comput. Accessibility, 2017, pp. 377-378.

[38] G. Sörös, F. Daiber, T. Weller, and I. Shenkar, "Cyclo: A personal bike coach through the glass," in Proc. SIGGRAPH ASIA Mobile Graph. Interactive Appl., 2013, Paper 99. 
[39] C. Ladha, N. Y. Hammerla, P. Olivier, and T. Plötz, "ClimbAX: Skill assessment for climbing enthusiasts," in Proc. ACM Int. Joint Conf. Pervasive Ubiquitous Comput., 2013, pp. 235-244.

[40] D. Spelmezan, "An investigation into the use of tactile instructions in snowboarding," in Proc. 14th Int. Conf. Hum.-Comput. Interact. Mobile Devices Services, 2012, pp. 417-426.

[41] D. Spelmezan, A. Schanowski, and J. Borchers, "Wearable automatic feedback devices for physical activities," in Proc. 4th Int. Conf. Body Area Netw., 2009, Paper 1.

[42] J. J. Chen, Y. F. Chung, C. P. Chang, C. T. King, and C. H. Hsu, "A wearable virtual coach for Marathon beginners," in Proc. 20th IEEE Int. Conf. Parallel Distrib. Syst., 2014, pp. 915-920.

[43] C. Feeken, M. Wasmann, W. Heuten, D. Ennenga, H. Müller, and S. Boll, "Climbingassist: Direct vibro-tactile feedback on climbing technique," in Proc. ACM Int. Joint Conf. Pervasive Ubiquitous Comput., Adjunct., 2016, pp. 57-60.

[44] C. Strohrmann, J. Seiter, Y. Llorca, and G. Tröster, "Can smartphones help with running technique?" Procedia Comput. Sci., vol. 19, pp. 902-907, 2013.

[45] P. H. Han, Y. S. Chen, Y. Zhong, H. L. Wang, and Y. P. Hung, "My Tai-Chi coaches: An augmented-learning tool for practicing Tai-Chi Chuan," in Proc. 8th Augmented Hum. Int. Conf., 2017, Paper 25.

[46] A. Weilenmann and L. E. Holmquist, "Hummingbirds go skiing: Using wearable computers to support social interaction," in Proc. 3rd Int. Symp. Wearable Comput., San Francisco, CA, USA, 1999, pp. 191-192.

[47] M. Hassan, F. Daiber, F. Wiehr, F. Kosmalla, and A. Krüger, "FootStriker: An EMS-based foot strike assistant for running," in Proc. ACM Conf. Interactive, Mobile, Wearable Ubiquitous Technol., 2017, vol. 1, Paper 2.

[48] M. Bächlin, K. Förster, and G. Tröster, "SwimMaster: A wearable assistant for swimmer," in Proc. 11th Int. Conf. Ubiquitous Comput., 2009, pp. $215-224$.

[49] A. Colley, J. Väyrynen, and J. Häkkilä, "Skiing in a blended virtuality: An in-the-wild experiment," in Proc. 19th Int. Acad. Mindtrek Conf., 2015, pp. 89-91.

[50] E. Niforatos, A. Fedosov, I. Elhart, and M. Langheinrich, "Augmenting skiers' peripheral perception," in Proc. ACM Int. Symp. Wearable Comput., 2017, pp. 114-121.

[51] A. Raina, T. G. Lakshmi, and S. Murthy, "CoMBaT: Wearable technology based training system for novice badminton players," in Proc. IEEE 17th Int. Conf. Adv. Learn. Technol., 2017, pp. 153-157.

[52] F. M. Valsted, C. V. Nielsen, J. Q. Jensen, T. Sonne, and M. M. Jensen, "Strive: Exploring assistive haptic feedback on the run," in Proc. 29th Aust. Conf. Comput.-Hum. Interact., 2017, pp. 275-284.

[53] C. D. Stewart, P. Traitor, and V. L. Hanson, "I'd Tap That! providing real time feedback on roller derby skills," in Proc. Extended Abstr. Hum. Factors Comput. Syst., 2014, pp. 2221-2226.

[54] M. Pan, S. Salvi, and E. Brady, "Designing auditory feedback from wearable weightlifting devices," in Proc. Extended Abstr. Hum. Factors Comput. Syst., 2018, Paper LBW561.

[55] E. Mencarini, C. Leonardi, A. De Angeli, and M. Zancanaro, "Design opportunities for wearable devices in learning to climb," in Proc. 9th Nordic Conf. Hum.-Comput. Interact., 2016, Paper 48.

[56] S. Bogers, C. Megens, and S. Vos, "Design for balanced engagement in mixed level sports teams," in Proc. SIGCHI Conf. Extended Abstr. Hum. Factors Comput. Syst., 2017, pp. 994-1002.

[57] V. G. Motti and K. Caine, "Smart wearables or dumb wearables?: Understanding how context impacts the UX in wrist worn interaction," in Proc. 34th ACM Int. Conf. Des. Commun., 2016, Paper 10.

[58] C. Thomas et al.., "The voice of the people: A system for enriching Olympic experiences," in Proc. Extended Abstr. Hum. Factors Comput. Syst., 2004, pp. 1695-1699.

[59] J. Häkkilä, M. Alhonsuo, L. Virtanen, J. Rantakari, A. Colley, and T. Koivumäki, "MyData approach for personal health - A service design case for young athletes," in Proc. 49th Hawaii Int. Conf. Syst. Sci., 2016, pp. 3493-3502.

[60] H. Havlucu, T. Eskenazi, B. Akgün, M. C. Onbaşlı, A. Coşkun, and Ö. Özcan, "Flow state feedback through sports wearables: A case study on tennis," in Proc. Des. Interact. Syst. Conf., 2018, pp. 1025-1039.

[61] T. Franke, C. Pieringer, and P. Lukowicz, "How should a wearable rowing trainer look like? A user study," in Proc. 15th Annu. Int. Symp. Wearable Comput., 2011, pp. 15-18.

[62] F. Kosmalla, F. Wiehr, F. Daiber, A. Krüger, and M. Löchtefeld, "ClimbAware - Investigating perception and acceptance of wearables in rock climbing," in Proc. SIGCHI Conf. Hum. Factors Comput. Syst., 2016, pp. 1097-1108.
[63] M. R. Bice, J. W. Ball, and S. McClaran, "Technology and physical activity motivation," Int. J. Sport Exercise Psychol., vol. 14, no. 4, pp. 295-304, 2016.

[64] P. Carrington, K. Chang, H. Mentis, and A. Hurst, “'But, I don't take steps': Examining the inaccessibility of fitness trackers for wheelchair athletes," in Proc. 17th Int. ACM SIGACCESS Conf. Comput. Accessibility, 2015, pp. 193-201.

[65] F. Curmi, M. A. Ferrario, and J. Whittle, "Biometric data sharing in the wild: Investigating the effects on online sports spectators," Int. J. Hum. Comput. Stud., vol. 105, pp. 56-67, Sep. 2017.

[66] T. Fritz, E. M. Huang, G. C. Murphy, and T. Zimmermann, "Persuasive technology in the real world: A study of long-term use of activity sensing devices for fitness," in Proc. SIGCHI Conf. Hum. Factors Comput. Syst., 2014, pp. 487-496.

[67] S. Gradl, B. M. Eskofier, D. Eskofier, C. Mutschler, and S. Otto, "Virtual and augmented reality in sports: an overview and acceptance study," in Proc. ACM Int. Joint Conf. Pervasive Ubiquitous Comput., Adjunct, 2016, pp. 885-888.

[68] X. Gui, Y. Chen, C. Caldeira, D. Xiao, and Y. Chen, "When fitness meets social networks: Investigating fitness tracking and social practices on WeRun," in Proc. CHI Conf. Hum. Factors Comput. Syst., 2017, pp. $1647-$ 1659 .

[69] H. Havlucu, I. Bostan, A. Coskun, and Ö. Özcan, "Understanding the lonesome tennis players: Insights for future wearables," in Proc. Extended Abstr. Hum. Factors Comput. Syst., 2017, pp. 1678-1685.

[70] H. Issa, A. Shafaee, S. Agne, S. Baumann, and A. Dengel, "User-sentiment based evaluation for market fitness trackers-evaluation of Fitbit one, Jawbone Up and Nike+ Fuelband based on amazon.com customer reviews," in Proc. ICT4AgeingWell, 2015, pp. 171-179.

[71] M. H. Jarrahi, N. Gafinowitz, and G. Shin, "Activity trackers, prior motivation, and perceived informational and motivational affordances," Pers. Ubiquitous Comput., vol. 22, no. 2, pp. 433-448, 2018.

[72] D. S. C. Kreitzberg, S. L. Dailey, T. M. Vogt, D. Robinson, and Y. Zhu, "What is your fitness tracker communicating?: Exploring messages and effects of wearable fitness devices," Qualitative Res. Commun., vol. 17, no. 1, pp. 93-101, 2016

[73] P. Nurkka, "Customization in long-term use: The case of the sports watch," in Proc. 15th Int. Conf. Mobile Ubiquitous Multimedia, 2016, pp. 5-10.

[74] M. Patel and A. A. O'Kane, "Contextual influences on the use and non-use of digital technology while exercising at the gym," in Proc. 33rd Annu. ACM Conf. Hum. Factors Comput. Syst., 2015, pp. 2923-2932.

[75] A. Rapp and L. Tirabeni, "Personal informatics for sport: Meaning, body, and social relations in amateur and elite athletes," ACM Trans. Comput. Hum. Interact., vol. 25, no. 3, 2018, Art. no. 16.

[76] P. Reyes-Mercado, "Adoption of fitness wearables: Insights from partial least squares and qualitative comparative analysis," J. Syst. Inf. Technol., vol. 20, no. 1, pp. 103-127, 2018.

[77] J. Stragier, T. Evens, and P. Mechant, "Broadcast yourself: An exploratory study of sharing physical activity on social networking sites," Media Int. Australia, vol. 155, no. 1, pp. 120-129, 2015.

[78] J. Tholander and S. Nylander, "Snot, sweat, pain, mud, and snow: Performance and experience in the use of sports watches," in Proc. SIGCHI Conf. Hum. Factors Comput. Syst., 2015, pp. 2913-2922.

[79] D. Malcolm, "Motivation," The SAGE Dictionary of Sport Studies. London, U.K.: Sage, 2008, pp. 172-173.

[80] P. M. Pedersen, P. C. Laucella, K. S. Miloch, and L. W. Fielding, "The juxtaposition of sport and communication: Defining the field of sport communication," Int. J. Sport Manage. Marketing, vol. 2, no. 3, pp. 193-207, 2007.

[81] L. E. Dunne et al., "The social comfort of wearable technology and gestural interaction," in Proc. Annu. Int. Conf. IEEE Eng. Med. Biol. Soc., 2014 pp. 4159-4162.

[82] C. Harrison, B. Y. Lim, A. Shick, and S. E. Hudson, "Where to locate wearable displays? Reaction time performance of visual alerts from tip to toe," in Proc. SIGCHI Conf. Hum. Factors Comput. Syst., 2009, pp. $941-$ 944.

[83] R. A. Schmidt, T. D. Lee, C. Winstein, G. Wulf, and H. N. Zelaznik, Motor Control and Learning: A Behavioral Emphasis. Champaign, IL, USA: Human Kinetics, 2018.

[84] E. Phillips, D. Farrow, K. Ball, and R. Helmer, "Harnessing and understanding feedback technology in applied settings," Sports Med., vol. 43, no. 10, pp. 919-925, 2013.

[85] D. E. Young, R. A. Schmidt, and T. D. Lee, "Skill learning: Augmented feedback," in International Encyclopedia of Ergonomics and Human Factors, W. Karwowski, Ed. London, U.K.: Taylor \& Francis, 2006, pp. 558-561. 
[86] D. Gill, L. Williams, and E. Reifsteck, Psychological Dynamics of Sport and Exercise. Champaign, IL, USA: Human Kinetics, 2017.

[87] R. E. Smith, "Toward a cognitive-affective model of athletic burnout," $J$. Sport Psychol., vol. 8, no. 1, pp. 36-50, 1986.

[88] A. R. Nicholls and R. C. Polman, "Coping in sport: A systematic review," J. Sport Sci., vol. 25, no. 1, pp. 11-31, 2007.

[89] J. A. Roduit and R. Gaehwiler, "Ethics and enhancement in sport: Becoming the fastest (human?) being," Sport Soc., vol. 21, no. 4, pp. 713-719, 2018.

[90] Ö. Simsekoglu and C. A. Klöckner, "The role of psychological and sociodemographical factors for electric bike use in Norway," Int. J. Sustain. Transp., vol. 13, pp. 1-9, 2018.

[91] S. Lukosch and K. Kunze, Eds., Proc. 1st Superhuman Sports Des. Challenge, 1st Int. Symp. Amplifying Capabilities Competing Mixed Realities, 2018.

[92] G. Aloi et al., "Enabling IoT interoperability through opportunistic smartphone-based mobile gateways," J. Netw. Comput. Appl. Vol., vol. 81, pp. 74-84, 2017.

[93] A. Singh, N. Bianchi-Berthouze, and A. C. Williams, "Supporting everyday function in chronic pain using wearable technology," in Proc. CHI Conf. Human Factors Comput. Syst., New York, NY, USA, 2017, pp. 39033915.

[94] A. Vasconcelos, F. Nunes, A. Carvalho, and C. Correia, "Mobile, exerciseagnostic, sensor-based serious games for physical rehabilitation at home," in Proc. 12th Int. Conf. Tangible, Embedded, Embodied Interact., New York, NY, USA, 2018, pp. 271-278.



Eleonora Mencarini received the Ph.D. degree in computer science from the University of Trento, Trento, Italy, in 2018.

She is currently a Postdoctoral Research Fellow with Fondazione Bruno Kessler, Trento, Italy. Her research field is human-computer interaction and her main interests in the field are embodied interaction, co-design, and wearable devices.

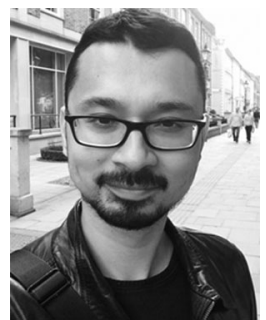

Amon Rapp received the Ph.D. degree in sciences of language and communication from the University of Torino in 2015.

$\mathrm{He}$ is a Research Fellow with the Computer Science Department, University of Torino, Torino, Italy, where he leads the Smart Personal Technology Lab at ICxT. His scientific research is situated within the area of human-computer interaction.

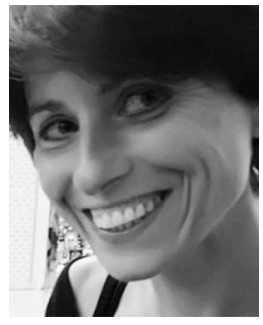

Lia Tirabeni received the Ph.D. degree in sociology from the University of Torino in 2014.

She is a Research Fellow with the Department of Cultures, Politics, and Society, University of Torino, Torino, Italy. Her research mainly concerns organization science and the way technology affects work practices and organizations.

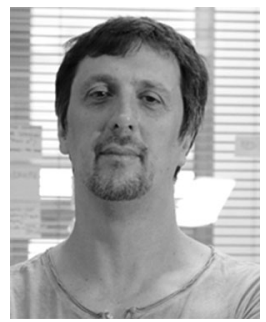

Massimo Zancanaro received the Laurea degree in computer science at the University of Milano in 1992. is a Full Professor of computer science with the Department of Psychology and Cognitive Science, University of Trento, Trento, Italy, and the Head of the i3-Intelligent Interfaces and Interaction-research unit at Fondazione Bruno Kessler, Trento, Italy. His research interests are in the field on human-computer interaction. 